\title{
¿Estamos cerrando muchos forámenes ovales permeables en Colombia? Foramen ovale permeable, aneurisma del septum interauricular y riesgo de ataque cerebrovascular criptogénico recurrente. Revisión sistemática de la literatura
}

\author{
Ángel Alberto García Peña ${ }^{a, *}$ y Jaime Alberto Rodríguez ${ }^{\mathrm{b}}$ \\ a Unidad de Cardiología, Hospital Universitario de San Ignacio. Pontificia Universidad Javeriana, Bogotá, Colombia \\ b Unidad de Cardiología, Fundación Cardiovascular de Colombia, Floridablanca, Colombia
}

Recibido el 8 de octubre de 2015; aceptado el 18 de abril de 2016

Disponible en Internet el 20 de junio de 2016

\section{PALABRAS CLAVE \\ Ictus; \\ Comunicación \\ interauricular; \\ Factores de riesgo; \\ Embolización}

\begin{abstract}
Resumen
Introducción: el foramen ovale permeable y el aneurisma del septum interauricular, son hallazgos frecuentes en la población general. En los últimos años múltiples estudios han sugerido la asociación entre estas entidades y el accidente cerebrovascular, de origen criptogénico como consecuencia de embolia paradójica, en especial en la población menor de 55 años, que ha estimulado el desarrollo de dispositivos de cierre percutáneo para este tipo de defectos, con el objetivo de reducir la recurrencia de eventos isquémicos cerebrales, sin que se hayan podido demostrar claramente estos beneficios.

Objetivo: realizar una revisión sistemática de la literatura para evaluar el riesgo de recurrencia y beneficio de esta intervención.

Resultados: Se obtuvieron 1.208 artículos para evaluación, con un total de 11 artículos incluidos para el análisis posterior a la selección, evaluación y aplicación de criterios de inclusión y exclusión, encontrando 3 ensayos clínicos y 8 revisiones sistemáticas y/o metaanálisis.

Conclusiones: En pacientes adultos con ataque cerebrovascular isquémico o accidente isquémico transitorio de origen criptogénico a quienes se les ha documentado mediante ecocardiografía transesofágica la presencia de foramen oval permeable, no existe beneficio en la reducción del riesgo de recurrencia de ataque cerebrovascular, accidente isquémico transitorio o eventos neurológicos, en comparación con la terapia médica convencional.

(c) 2016 Sociedad Colombiana de Cardiología y Cirugía Cardiovascular. Publicado por Elsevier España, S.L.U. Este es un artículo Open Access bajo la licencia CC BY-NC-ND (http:// creativecommons.org/licenses/by-nc-nd/4.0/).
\end{abstract}

\footnotetext{
* Autor para correspondencia.

Correo electrónico: angel.garcia@javeriana.edu.co (Á.A. García Peña).
} 


\section{KEYWORDS}

Stroke;

Atrial septal defect;

Risk factors;

Embolization
Are many patent foramen ovale closure procedures being performed in Colombia? Patent foramen ovale, atrial septal aneurysm and the risk of recurring cryptogenic stroke. Systematic review of literature

\begin{abstract}
Introduction: Patent foramen ovale and atrial septal aneurysm are frequent findings in the average population. Over the last years multiple studies have suggested there is an association between both entities and cerebrovascular accidents of cryptogenic origin as a consequence of paradoxical embolism, specially among population under age 55 , which has stimulated the development of devices for percutaneous closure of these defects with the goal of reducing recurring cerebral ischemic events, without a clear demonstration of their efficacy.

Motivation: To perform a systematic review of literature to assess the risk of recurrence and benefits of this surgery.

Results: 1,208 articles were collected for this evaluation, with a total of 11 papers included in the analysis following selection, assessment and application of selection criteria. 3 of them were clinical trial and 8 were systematic reviews or meta-analysis.

Conclusions: In adult patients with cryptogenic cerebrovascular ischemic or transient ischemic attacks with patent foramen ovale documented via transthoracic echocardiogram no decrease has been found in the risk of recurrence of cerebrovascular attacks, transient ischemic accidents or neurological events in comparison with traditional medical therapy.

( $) 2016$ Sociedad Colombiana de Cardiología y Cirugía Cardiovascular. Published by Elsevier España, S.L.U. This is an open access article under the CC BY-NC-ND license (http:// creativecommons.org/licenses/by-nc-nd/4.0/).
\end{abstract}

\section{Introducción}

El foramen ovale permeable y el aneurisma del septum interauricular, son hallazgos comunes en la población general, de hecho se estima una prevalencia del 25 y el $2,5 \%$ respectivamente ${ }^{1,2}$. En los últimos años, múltiples estudios han sugerido la asociación entre foramen oval permeable, aneurisma del septum interauricular y ataque cerebrovascular de origen criptogénico como consecuencia de embolia paradójica, especialmente en la población menor de 55 años; sin embargo, no se ha demostrado de forma contundente una fuerte relación de causalidad ${ }^{3,4}$. En las últimas décadas, el desarrollo de dispositivos de cierre percutáneo para este tipo de defectos ha tenido un desarrollo vertiginoso y su uso se ha masificado, en ocasiones con una indicación "off-label", sin que se haya demostrado con exactitud una reducción en la recurrencia de eventos isquémicos cerebrales ${ }^{5-8}$. La evidencia sobre las indicaciones de cierre percutáneo con dispositivo del foramen oval permeable y aneurisma del septum interauricular, y la reducción del riesgo de nuevos eventos isquémicos al comparar con la terapia médica, es controversial. En la práctica médica diaria se indica su cierre cuando existe asociación con ataque cerebrovascular pese a que la evidencia obtenida en algunos metaanálisis ${ }^{9,10}$, sugiere mayor morbilidad con la alternativa de cierre percutáneo y puede ser inferior en la capacidad de reducir este riesgo frente al manejo médico. Por esta razón, a través de una revisión sistemática, se propone proceder a la búsqueda, análisis, evaluación y síntesis de la evidencia disponible sobre el riesgo de foramen oval permeable-aneurisma del septum interauricular y ataque cerebrovascular criptogénico, con el fin de evaluar diferencias en definición, reducción del riesgo y beneficio real del cierre.

\section{Marco teórico y estado del arte}

El foramen oval permeable es una comunicación entre las dos aurículas, indispensable durante la vida fetal, que permite el paso de sangre oxigenada procedente de la placenta hacia la circulación sistémica. Está formado por la sobreposición incompleta del septum secundum y el remanente superior del septum primum, cuyo cierre funcional sucede inmediatamente después del parto, como consecuencia de los cambios en las presiones de las cavidades izquierdas y derechas. En los primeros años de vida estas hojas se fusionan dando como resultado la fosa oval. Estudios realizados mediante ecocardiografía y autopsias describen que una de cada 4 personas de la población general, es portadora de foramen oval el cual puede permeabilizarse de manera espontánea, fenómeno que conlleva un cortocircuito de derecha a izquierda y genera embolia paradójica ${ }^{11}$. Este fue descrito en 1881 por Zahn ${ }^{12}$ en un estudio postmortem de una mujer con trombo uterino y a partir de allí se estableció su relación con el ataque cerebrovascular. Si bien la frecuencia de foramen oval permeable disminuye con la edad, su tamaño incrementa con ella ${ }^{13}$.

Se considera que hay aneurisma del septum interauricular cuando en forma parcial o total, este tabique se encuentra adelgazado y protruye hacia alguna de las aurículas con el ciclo respiratorio. Dicho desplazamiento debe ser superior a $15 \mathrm{~mm}$. Un tercio de estos pacientes tiene además foramen oval permeable y es más grande en aquellos con aneurisma del septum interauricular.

De otro lado, se estima que 800.000 personas presentan ataque cerebrovascular cada año en Estados Unidos, de los cuales 610.000 son un primer episodio y 185.000 son considerados recurrentes ${ }^{14}$. Aproximadamente, un 25 a un $40 \%$ de estos se clasifican como criptogénicos o de causa 
no clara y es aquí en donde el foramen oval permeable es más frecuente que en la población general o en el grupo de pacientes con ataque cerebrovascular de causa conocida ${ }^{15}$.

$\mathrm{Si}$ bien existen algunos estudios observacionales que muestran un incremento del riesgo embólico en los pacientes que cursan simultáneamente con aneurisma del septum interauricular y foramen oval permeable, otros no han demostrado resultados similares ${ }^{2}$.

La asociación entre foramen oval permeable-aneurisma del septum interauricular y ataque cerebrovascular criptogénico es heterogénea debido a la frecuencia diversa de foramen oval permeable en la población general, al riesgo diferencial de evento cerebrovascular en distintas poblaciones, a las diferencias en las características demográficas y a las múltiples definiciones utilizadas en los estudios clínicos.

Otro factor a tener en cuenta es el método diagnóstico utilizado, en cuyo caso la ecocardiografía 2D transtorácica o transesofágica y el Doppler transcraneal con uso de contraste de solución salina agitada, y recientemente el desarrollo de nuevos métodos diagnósticos a través de la ecocardiografía 3 $\mathrm{D}$ y la resonancia magnética cardiaca son los más utilizados y estandarizados en la práctica clínica. Así mismo, existe importante variabilidad de los criterios diagnósticos utilizados en los diferentes estudios ${ }^{16}$. Se estima que el ecocardiograma transtorácico posee menor sensibilidad que el transesofágico para la detección del foramen oval permeable, pero a su vez la sedación utilizada en los estudios ecocardiográficos transesofágicos podría disminuir la eficacia de la maniobra de Valsalva requerida para demostrar el cortocircuito ${ }^{16}$. El criterio utilizado para el diagnóstico de aneurisma del septum interauricular podría variar desde una excursión total del septum interauricular de 10 a $15 \mathrm{~mm}$, hasta una excursión fásica de $15 \mathrm{~mm}$ en cualquier dirección ${ }^{17}$.

El cierre percutáneo del foramen oval permeable en pacientes con ataque cerebrovascular criptogénico se realiza desde 1992 hasta la fecha, con una indicación off-label, dado que los estudios clínicos realizados (CLOSURE 1, RESPECT, PC Trial ${ }^{18-20}$ ) no han demostrado superioridad en comparación con el manejo médico.

En conclusión, el cierre del foramen oval permeable continúa siendo objeto de debate y no hay evidencia clara que respalde su cierre sistemático en los casos de ataque cerebrovascular criptogénico.

\section{Pregunta de investigación}

En individuos mayores de 18 años con foramen oval, ¿cuál es el riesgo de presentar un nuevo (recurrencia) ataque cerebrovascular de origen criptogénico?

\section{Definiciones}

1. Ataque cerebrovascular-evento cerebrovascular y accidente isquémico transitorio: equivalen a evento cerebrovascular, que hace referencia en ambos casos (ataque cerebrovascular-evento cerebrovascular) a un evento súbito que ocurre cuando el suministro de sangre de una zona del cerebro se interrumpe de manera definitiva (ataque cerebrovascular-evento cerebrovascular) o transitoria, en cuyo caso se denomina accidente isquémico transitorio. Está causado por cualquier anomalía en el cerebro como resultado de un proceso patológico de los vasos sanguíneos, incluyendo oclusión de la luz por embolia o trombosis, ruptura de un vaso, alteración de la permeabilidad de la pared de este o aumento de la viscosidad $u$ otro cambio en la calidad de la sangre que fluye a través de los vasos cerebrales.

2. Ataque cerebrovascular criptogénico: se define como aquel que ocurre cuando el suministro de sangre de una zona del cerebro se interrumpe en forma temporal o definitiva sin que después de los estudios completos quede dentro de alguna de las características diagnósticas (no es posible establecer la causa).

3. Defectos del septum interauricular (interatrial): anomalías del desarrollo en cualquier parte del tabique interatrial que generan comunicaciones anormales entre las aurículas. Su clasificación se basa en la localización de la comunicación y en los tipos de fusión incompleta de los tabiques interatriales con los cojinetes endocárdicos en el corazón del feto.

4. Tipos de defectos: la clasificación de los tipos de defecto se basa en la localización de la comunicación y en los tipos de fusión incompleta de los tabiques interatriales con los cojinetes endocárdicos en el corazón del feto. Dentro de ellos están el foramen ovale permeable y la comunicación interauricular con sus diferentes tipos (tipo ostium, ostium primum, seno venoso y aurícula única o aurícula común). Para efectos de este trabajo, solo se hará énfasis en el foramen oval permeable y en el aneurisma del septum interauricular.

\section{Metodología}

Se realizó una revisión sistemática de la literatura con el objetivo de determinar el riesgo de accidente cerebrovascular criptogénico recurrente asociado con la presencia de foramen oval permeable y aneurisma del septum interauricular y además se tuvieron en cuenta los aspectos técnicos relacionados con el diagnóstico de estos mediante ecocardiografía transesofágica.

La población objeto del estudio fueron adultos con foramen oval permeable y aneurisma del septum interauricular. El desenlace seleccionado fue ataque cerebrovascular diagnosticado por personal médico con base en hallazgos clínicos e imagenológicos de acuerdo con los criterios de clasificación ICD (International Statistical Classification of Diseases and Related Health Problems), versión 10, códigos 160-169. Se realizó una búsqueda en las diferentes bases de datos a partir de 1966 a mayo de 2015. Los términos Mesh utilizados fueron: atrial septum, atrial septum aneurysm, atrial septal defects, stroke, embolism, transesophageal echocardiography, $3 \mathrm{~d}$ transesophageal echocardiography, transesophageal echocardiography $3 \mathrm{~d}$, transesophageal echocardiography stroke, risk. La búsqueda se limitó a humanos e idioma inglés y español.

Se emplearon las bases de datos: EMBASE, Medline, SciELO, Cochrane, Clinical evidence, Dynamed, registro de experimentos clínicos de Cochrane y LILACS, así como las 
guías de práctica clínica propuestas por la ASE/EAE (American Society of Echocardiography and European Association of Echocardiography) y sus referencias, revisiones sistemáticas indexadas, metaanálisis y artículos no publicados, y registros de trabajos en congresos sugeridos por expertos, temáticos y metodológicos.

Se incluyeron ensayos clínicos (aleatorizados, abiertos y cruzados), revisiones sistemáticas de la literatura y metaanálisis.

Se excluyeron reportes de casos, editoriales, cohortes y casos de controles (inicialmente se aplicó como criterios de exclusión; de no encontrar literatura, este aspecto fue removido e incluido en criterios de selección).

Se hicieron las diferentes búsquedas a fin de evaluar la literatura disponible en las bases de datos en mención. Posteriormente, de forma pareada se procedió a realizar la selección de los artículos por título y posteriormente por resumen (abstract). Para la evaluación de la calidad de estudios primarios, revisiones sistemáticas y metaanálisis, se obtuvieron los artículos en texto completo y se evaluaron de forma independiente por los miembros del equipo de trabajo, utilizando los criterios propuestos por Gordon Guyatt y Drummond Rennie en la Users' Guides to the Medical Literature ${ }^{21}$. Las discrepancias entre los revisores fueron resueltas por consenso al interior del grupo. Posteriormente, se procedió a realizar extracción de los datos para el respectivo análisis.

\section{Resultados}

Se procedió a ejecutar la búsqueda, selección y evaluación de los estudios según protocolo propuesto, encontrando un total de 1.208 artículos, de los cuales se seleccionaron por título y resumen 58 , se eliminaron 31 duplicados, para un total de 27 artículos que se evaluaron en versión texto completo. La selección final para análisis constó de 11 artículos (fig. 1). Así mismo, en la tabla 1 se resume la evaluación de los demás artículos encontrados.

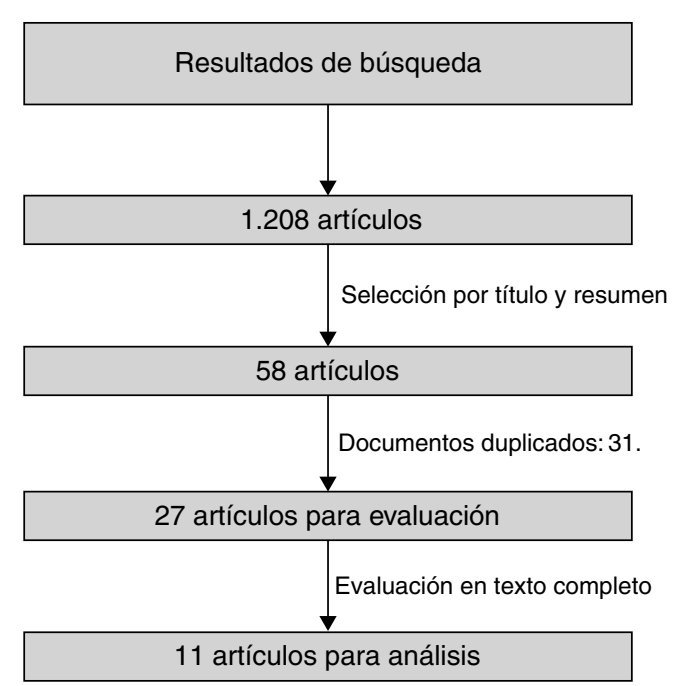

Figura 1 Selección de artículos.

\section{Descripción de los estudios}

Dentro de los artículos incluidos para el análisis final, se encuentran 3 ensayos clínicos y 8 revisiones sistemáticas o metaanálisis. A continuación se realiza una breve descripción de cada uno de los artículos.

Furlan et al. ${ }^{18}$ en 2012, presentó el CLOSURE I, un estudio multicéntrico, aleatorizado de cierre percutáneo con dispositivo Starflex ${ }^{\circledR}$ vs. terapia médica, que incluyó pacientes de 18 a 60 años, con antecedente de ataque cerebrovascular criptogénico o accidente isquémico transitorio en los 6 meses previos al ingreso al estudio, con documentación de foramen oval permeable mediante ecocardiograma transesofágico y aplicación de burbujas (inyección de solución salina agitada con demostración de cortocircuito de derecha a izquierda a nivel auricular durante las maniobras de Valsalva). Se excluyeron pacientes con identificación de cualquier causa potencial de ataque cerebrovascular, disfunción ventricular izquierda, aneurisma ventricular y fibrilación auricular. La asignación se realizó 1:1; al grupo asignado a dispositivo se le suministró terapia antiplaquetaria (clopidogrel $75 \mathrm{mg}$ por 6 meses y ácido acetil salicílico 81-325 mg por 2 años); al grupo asignado a tratamiento médico se le suministró terapia antitrombótica (warfarina con INR objetivo 2-3, ácido acetil salicílico $81-325 \mathrm{mg}$ o los dos, a discreción del grupo tratante). El desenlace primario fue un desenlace compuesto que incluyó ataque cerebrovascular, accidente isquémico transitorio durante los 2 años de seguimiento, muerte por cualquier causa en los primeros 30 días o muerte por causa neurológica entre el día 31 y los 2 años de seguimiento. Como desenlaces secundarios se incluyó sangrado mayor, muerte por cualquier causa, ataque cerebrovascular, accidente isquémico transitorio o eventos neurológicos transitorios de causa incierta. Se incluyeron en total 909 pacientes entre los años 2003 y 2008, en 87 centros en Estados Unidos y Canadá. 447 fueron asignados a cierre con dispositivo y 462 a terapia médica. Se logró implantación del dispositivo en el $89,4 \%$, siendo efectivo en el $86,1 \%$; el porcentaje de pacientes con aneurisma del septum interauricular fue de $37,6 \%$ para el grupo de dispositivo vs. $35,7 \%$ para el grupo de terapia médica. No existieron diferencias significativas en las características demográficas o perfil de riesgo de los pacientes incluidos. El análisis fue presentado por protocolo y por intención de tratar, sin encontrar diferencias significativas entre los grupos; en el análisis por intención a tratar el desenlace primario se presentó en el $5,5 \%$ de los pacientes asignados al grupo de dispositivo 5,5 vs. $6,8 \%$ en el grupo asignado a terapia médica con hazard ratio (HR) 0,78, intervalo de confianza del $95 \%$ (IC 95) $(0,45-1,35), p=0,37$ y ataque cerebrovascular 2,9 vs. 3,1\% (HR: 0,90 IC 95: 0,41-1,98; $p=0,79$ ). En el análisis por protocolo se encontró para el desenlace primario 5,8 vs. $7,7 \%$ (HR 0,74; IC 95: 0,42-1,29; $p=0,28$ y para ataque cerebrovascular 3,2 vs. $3,5 \%$ (HR 0,91; IC 95: 0,41-1,99; $p=0,80$ ). Como eventos adversos en el grupo de dispositivo se documentaron complicaciones vasculares mayores en el 3,2\% de la población, sangrado mayor en el 2,6\% en el grupo de dispositivo vs. $1,1 \%$ en el grupo de terapia médica, $p=0,11$ y fibrilación auricular de novo en el 5,7\%, siendo esta más frecuente en los primeros 30 días posterior al implante del dispositivo. En la discusión acerca de los ataques cerebrovascular o isquémico transitorio recurrentes, los autores 
Tabla 1 Resumen de la evaluación de los artículos

\begin{tabular}{|c|c|c|c|c|c|}
\hline Referencia & Título & Tipo & $J R$ & AG & $A / R$ \\
\hline 22 & Atrial septal aneurysm in adult patients & EC & - & - & Rechazado \\
\hline 19 & $\begin{array}{l}\text { Closure or medical therapy for cryptogenic stroke with patent } \\
\text { foramen ovale }\end{array}$ & EC & + & + & Aprobado \\
\hline 18 & $\begin{array}{l}\text { Closure or medical therapy for cryptogenic stroke with patent } \\
\text { foramen ovale }\end{array}$ & EC & + & + & Aprobado \\
\hline 23 & $\begin{array}{l}\text { Device closure of patent foramen ovale versus medical therapy in } \\
\text { cryptogenic stroke a systematic review and meta-analysis }\end{array}$ & $\begin{array}{l}\text { RS, } \\
M- \\
\text { A }\end{array}$ & + & + & Aprobado \\
\hline 10 & $\begin{array}{l}\text { Is transcatheter closure better than medical therapy for } \\
\text { cryptogenic stroke with patent foramen ovale? A meta-analysis } \\
\text { of randomised trials }\end{array}$ & $\begin{array}{l}\text { RS, } \\
M- \\
\text { A }\end{array}$ & + & + & Aprobado \\
\hline 24 & $\begin{array}{l}\text { Long term follow up after percutaneous closure of PFO in } 357 \\
\text { patients with paradoxical embolism: Difference in occlusion } \\
\text { systems and influence of atrial septum aneurysm }\end{array}$ & EC & - & - & Rechazado \\
\hline 25 & $\begin{array}{l}\text { Long-term results of a randomized trial comparing three } \\
\text { different devices for percutaneous closure of a patent foramen } \\
\text { ovale }\end{array}$ & $\begin{array}{l}\text { RS, } \\
M- \\
\text { A }\end{array}$ & - & - & Rechazado \\
\hline 26 & $\begin{array}{l}\text { Meta-analysis of net long-term benefit of different therapeutic } \\
\text { strategies in patients with cryptogenic stroke and patent } \\
\text { foramen ovale }\end{array}$ & $\begin{array}{l}\text { RS, } \\
M- \\
\text { A }\end{array}$ & - & - & Rechazado \\
\hline 5 & $\begin{array}{l}\text { Meta-analysis of transcatheter closure versus medical therapy } \\
\text { for patent foramen ovale in prevention of recurrent neurological } \\
\text { events } \\
\text { after presumed paradoxical embolism }\end{array}$ & $\begin{array}{l}\text { RS, } \\
M- \\
\text { A }\end{array}$ & - & - & Rechazado \\
\hline 7 & $\begin{array}{l}\text { Patent foramen ovale closure and medical treatments for } \\
\text { secondary stroke prevention a systematic review of observational } \\
\text { and randomized evidence }\end{array}$ & $\begin{array}{l}\mathrm{RS} \\
\mathrm{M}- \\
\mathrm{A}\end{array}$ & - & - & Rechazado \\
\hline 27 & $\begin{array}{l}\text { Patent foramen ovale closure vs medical therapy for stroke } \\
\text { prevention: meta-analysis of randomized trials and review of } \\
\text { heterogeneity in meta-analyses }\end{array}$ & $\begin{array}{l}\text { RS, } \\
\text { M- } \\
\text { A }\end{array}$ & + & + & Aprobado \\
\hline 28 & $\begin{array}{l}\text { Patent foramen ovale transcatheter closure vs. medical therapy } \\
\text { on recurrent vascular events: a systematic review and } \\
\text { meta-analysis of randomized controlled trials }\end{array}$ & $\begin{array}{l}\text { RS, } \\
\text { M- } \\
\text { A }\end{array}$ & + & + & Aprobado \\
\hline 29 & $\begin{array}{l}\text { Patent foramen ovale, ischemic stroke and migraine: systematic } \\
\text { review and stratified meta-analysis of association studies }\end{array}$ & $\begin{array}{l}\mathrm{RS}, \\
\mathrm{M}- \\
\mathrm{A}\end{array}$ & - & - & Rechazado \\
\hline 30 & $\begin{array}{l}\text { Percutaneous closure of patent foramen ovale for cryptogenic } \\
\text { stroke: A meta-analysis of randomized controlled trials }\end{array}$ & $\begin{array}{l}\mathrm{RS} \\
\mathrm{M}- \\
\mathrm{A}\end{array}$ & + & + & Aprobado \\
\hline 20 & $\begin{array}{l}\text { Percutaneous closure of patent foramen ovale in cryptogenic } \\
\text { embolism }\end{array}$ & EC & + & + & Aprobado \\
\hline 31 & Percutaneous closure versus medical therapy alone & $\begin{array}{l}\text { RS, } \\
M- \\
\text { A }\end{array}$ & + & + & Aprobado \\
\hline 32 & $\begin{array}{l}\text { PFO closuRE and Cryptogenlc StrokE (PRECISE) registry: a } \\
\text { multi-center, international registry }\end{array}$ & $\begin{array}{l}\mathrm{RS} \\
\mathrm{M}- \\
\mathrm{A}\end{array}$ & - & - & Rechazado \\
\hline 33 & PFO Closure: CLOSURE. Subanálisis & EC & - & - & Rechazado \\
\hline 34 & $\begin{array}{l}\text { Recurrent cerebrovascular events associated with patent } \\
\text { foramen ovale, atrial septal aneurysm, or both }\end{array}$ & $\mathrm{RS}$ & - & - & Rechazado \\
\hline 35 & $\begin{array}{l}\text { Risk of stroke in patients with patent foramen ovale: an updated } \\
\text { meta-analysis of observational studies }\end{array}$ & $\begin{array}{l}\text { RS, } \\
M- \\
\text { A }\end{array}$ & - & - & Rechazado \\
\hline 36 & $\begin{array}{l}\text { Safety and efficacy of device closure for patent foramen ovale } \\
\text { for secondary prevention of neurological events: Comprehensive } \\
\text { systematic review and meta-analysis of randomized controlled } \\
\text { trials }\end{array}$ & $\begin{array}{l}\text { RS, } \\
M- \\
\text { A }\end{array}$ & + & + & Aprobado \\
\hline
\end{tabular}


Tabla 1 (continuación)

\begin{tabular}{|c|c|c|c|c|c|}
\hline Referencia & Título & Tipo & $J R$ & $A G$ & $A / R$ \\
\hline 37 & $\begin{array}{l}\text { Short and long term complications of device closure of atrial } \\
\text { septal defect and patent foramen ovale: meta-analysis of } 28,142 \\
\text { patients from } 203 \text { studies }\end{array}$ & $\begin{array}{l}\text { RS, } \\
M- \\
\text { A }\end{array}$ & - & - & Rechazado \\
\hline 38 & $\begin{array}{l}\text { Transcatheter patent foramen ovale closure versus medical } \\
\text { therapy for cryptogenic stroke: a meta-analysis of randomized } \\
\text { clinical trials }\end{array}$ & $\begin{array}{l}\text { RS, } \\
M- \\
\text { A }\end{array}$ & + & + & Aprobado \\
\hline 39 & $\begin{array}{l}\text { Transesophageal echocardiography in patients with cryptogenic } \\
\text { ischemic stroke: A systematic review }\end{array}$ & $\begin{array}{l}\text { RS, } \\
M- \\
A\end{array}$ & - & - & Rechazado \\
\hline 40 & $\begin{array}{l}\text { Transoesophageal echocardiography can help distinguish between } \\
\text { patients with "symptomatic" and "asymptomatic" patent } \\
\text { foramen ovale }\end{array}$ & EC & - & - & Rechazado \\
\hline 41 & $\begin{array}{l}\text { Updating the evidence on patent foramen ovale closure versus } \\
\text { medical therapy in patients with cryptogenic stroke: a systematic } \\
\text { review and comprehensive meta-analysis of 2,303 patients from } \\
\text { three randomised trials and 2,231 patients from } 11 \text { observational } \\
\text { studies. }\end{array}$ & $\begin{array}{l}\text { RS, } \\
M- \\
\text { A }\end{array}$ & - & - & Rechazado \\
\hline 42 & $\begin{array}{l}\text { Stroke prevention by percutaneous closure of patent foramen } \\
\text { ovale: a systematic review and meta-analysis. }\end{array}$ & $\begin{array}{l}\text { RS, } \\
M- \\
\text { A }\end{array}$ & - & - & Rechazado \\
\hline
\end{tabular}

RS: revisión sistemática, M-A: Meta-análisis. EC: Ensayo clínico. AG/JR evaluadores. A/R: aprobado-rechazado.

describen dentro de las posibles causas: fibrilación auricular de novo, coágulo en aurícula izquierda, infarto subcortical en presencia de factores de riesgo, ateroma del arco aórtico, migraña compleja y vasculitis, entre otras. Como conclusion, el cierre con dispositivo Starflex ${ }^{\circledR}$ en esta población, no muestra un beneficio adicional en comparación con la terapia médica, siendo evidente el incremento de los eventos adversos, en especial de fibrilación auricular de novo.

En 2013, Carroll $^{19}$, por su parte, presentó RESPECT Trial, un estudio multicéntrico, prospectivo, controlado, aleatorizado, abierto con adjudicación cegada de eventos finales, en el que se incluyeron pacientes de 18 a 60 años de edad, con ataque cerebrovascular isquémico de origen criptogénico en los 270 días previos a la aleatorización con documentación mediante ecocardiograma transesofágico de foramen oval permeable, y se excluyeron pacientes con otras causas de ataque cerebrovascular u otras fuentes de cardioembolia e hipercoagulabilidad. La asignación fue 1:1. En las medicaciones antitrombóticas permitidas estaban: aspirina, warfarina, clopidogrel y la combinación de aspirina y dipiridamol. En el grupo asignado a dispositivo se administró aspirina $81-325 \mathrm{mg} /$ día, más clopidogrel $75 \mathrm{mg} /$ día durante el primer mes posterior al implante del dispositivo y aspirina 81-325 mg/día por 6 meses; posterior a este momento, el uso de terapia antitrombótica quedaba a discreción del grupo tratante. El desenlace primario fue un punto final compuesto que incluyó: ataque cerebrovascular isquémico recurrente no fatal, ataque cerebrovascular isquémico o muerte. Se incluyeron un total de 980 pacientes entre los años 2003 a 2011, de los cuales 499 fueron asignados al grupo de cierre con dispositivo y 481 al grupo de terapia médica, por un periodo promedio de seguimiento de 2,1 años, durante el cual se registró pérdida del $17,2 \%$ de los pacientes en el grupo de terapia médica y $9,2 \%$ en el grupo de dispositivo. El dispositivo de cierre percutáneo utilizado fue Amplatzer PFO Occluder ${ }^{\circledR}$, el cual se logró implantar en el 99,1\% de los pacientes asignados a este grupo. La presencia de aneurisma del septum interauricular fue de $36,1 \%$ en el grupo asignado a dispositivo vs. $35,1 \%$ en el grupo asignado a terapia médica. No hubo diferencias significativas en las características demográficas o en el perfil de riesgo de la población incluida. Considerando la pérdida de pacientes y los análisis preespecificados, los autores presentan los resultados por protocolo y con intención a tratar en cohortes de tratamiento y seguimiento completas.

En el análisis por intención a tratar hubo una tasa de eventos (desenlace primario) de 0,66 por 100 pacientes/año en el grupo asignado a dispositivo vs. 1,38 por 100 pacientes/año en el grupo de terapia médica (HR 0,49; IC 95: $0,22-1,11 ; p=0,08)$. En el análisis por protocolo se registró una tasa de eventos (desenlace primario) de 0,46 por 100 pacientes/año en el grupo asignado a dispositivo vs. 1,3 por 100 pacientes/año en el grupo de terapia médica (HR 0,37 ; IC 95: 0,14-0,96; $p=0,03$ ). Sin embargo, en la discusión los autores describen las limitaciones de este análisis por el porcentaje de sujetos perdidos en el estudio, encontrando que podría existir un beneficio potencial en pacientes con algunas características de alto riesgo (tipo de shunt, perfil de riesgo, tamaño del ataque cerebrovascular); no obstante, dada la heterogeneidad de los datos, no fue posible establecer esta conclusión. Entre tanto, la tasa de eventos adversos fue del $23 \%$ en el grupo asignado a dispositivo vs. $21,6 \%$ en el de terapia médica, sin diferencias significativas entre ambos grupos. En conclusión, el cierre con dispositivo en esta población, no confiere un beneficio adicional al tratamiento médico convencional en términos de recurrencia de ataque cerebrovascular, accidente isquémico transitorio o muerte. 


\begin{tabular}{|c|c|c|c|c|c|c|c|c|c|c|}
\hline Estudio & Cohorte & $\mathrm{n}$ & $\begin{array}{l}\text { Edad } \\
\text { promedio }\end{array}$ & Hombres & $\begin{array}{l}\text { Aneurisma } \\
\text { Septum }\end{array}$ & $\begin{array}{l}\text { Años } \\
\text { seguimiento }\end{array}$ & Intervención & $\begin{array}{l}\text { Manejo } \\
\text { médico }\end{array}$ & $\begin{array}{l}\text { Desenlace } \\
\text { primario }\end{array}$ & Conclusión \\
\hline $\begin{array}{l}\text { Closure of patent } \\
\text { foramen ovale } \\
\text { versus medical } \\
\text { therapy after } \\
\text { cryptogenic } \\
\text { stroke. N Engl J } \\
\text { Med. } \\
\text { 2013;368:1092- } \\
\text { 100. RESPECT } \\
\text { Trial }^{19}\end{array}$ & $\begin{array}{l}\text { Prospectivo, } \\
\text { multicéntrico, } \\
\text { controlado, } \\
\text { aleatorizado, } \\
\text { abierto con } \\
\text { adjudicación } \\
\text { cegada de los } \\
\text { eventos finales }\end{array}$ & 980 & 45,9 años & $54,7 \%$ & $35,6 \%$ & $\begin{array}{l}2,6 \pm 2,0 \\
\text { años }\end{array}$ & $\begin{array}{l}\text { Amplatzer } \\
\text { PFO }^{\circledR} \text { occlu- } \\
\text { der + Aspirina } \\
\text { y } \\
\text { clopidogrel } \\
\text { por } 1 \text { mes } \\
\text { seguido de } \\
\text { aspirina }\end{array}$ & $\begin{array}{l}\text { Aspirina } \\
46,5 \% \\
\text { Warfarina } \\
25,2 \% \\
\text { Clopidogrel } \\
14 \% \\
\text { Aspirina } \\
+ \text { dipiridamol } \\
\text { 8,1\% } \\
\text { Aspirina } \\
+ \text { clopidogrel } \\
6,2 \%\end{array}$ & $\begin{array}{l}\text { Todas las } \\
\text { causas de } \\
\text { muerte; } \\
\text { recurrencia } \\
\text { de ECV } \\
\text { isquémico } \\
\text { fatal o no } \\
\text { fatal }\end{array}$ & $\begin{array}{l}\text { En el análisis } \\
\text { por intención a } \\
\text { tratar no hubo } \\
\text { beneficio } \\
\text { significativo } \\
\text { asociado con el } \\
\text { cierre de } \\
\text { foramen oval } \\
\text { permeable en } \\
\text { adultos que } \\
\text { tuvieron un } \\
\text { ECV isquémico } \\
\text { criptogénico. }\end{array}$ \\
\hline $\begin{array}{l}\text { Closure or medical } \\
\text { therapy for } \\
\text { cryptogenic } \\
\text { stroke with } \\
\text { patent foramen } \\
\text { ovale. N Engl J } \\
\text { Med. } \\
\text { 2012;366:991-9. } \\
\text { CLOSURE I trial }{ }^{18}\end{array}$ & $\begin{array}{l}\text { Prospectivo, } \\
\text { multicéntrico, } \\
\text { aletorizado, } \\
\text { abierto, } \\
\text { estudio de } \\
\text { superioridad } \\
\text { entre los } 2 \\
\text { grupos }\end{array}$ & 909 & 46 años & $51,8 \%$ & $36 \%$ & 2,0 años & $\begin{array}{l}\text { STARFlex }^{\circledR} \\
+ \text { aspirina ( } 2 \\
\text { años) y } \\
\text { clopidogrel } \\
\text { (6 meses) }\end{array}$ & $\begin{array}{l}\text { Aspirina, } \\
\text { warfarina o } \\
\text { aspirina y } \\
\text { warfarina (a } \\
\text { discreción } \\
\text { del médico } \\
\text { tratante) }\end{array}$ & $\begin{array}{l}\text { Compuesto } \\
\text { de ECV o } \\
\text { AIT, muerte } \\
\text { de cualquier } \\
\text { causa ( } 30 \\
\text { días), } \\
\text { muerte de } \\
\text { causa } \\
\text { neurológica } \\
\text { (31 días a } 2 \\
\text { años) }\end{array}$ & $\begin{array}{l}\text { En pacientes } \\
\text { con ECV } \\
\text { Criptogénico o } \\
\text { AIT que } \\
\text { tuvieron cierre } \\
\text { de foramen } \\
\text { oval permeable } \\
\text { con dispositivo, } \\
\text { el cierre no } \\
\text { ofrece mayor } \\
\text { beneficio que } \\
\text { la terapia } \\
\text { médica sola } \\
\text { para la } \\
\text { prevención de } \\
\text { ECV o AIT } \\
\text { recurrente. }\end{array}$ \\
\hline
\end{tabular}




\begin{tabular}{|c|c|c|c|c|c|c|c|c|c|c|}
\hline Estudio & Cohorte & $\mathrm{n}$ & $\begin{array}{l}\text { Edad } \\
\text { promedio }\end{array}$ & Hombres & $\begin{array}{l}\text { Aneurisma } \\
\text { Septum }\end{array}$ & $\begin{array}{l}\text { Años } \\
\text { seguimiento }\end{array}$ & Intervención & $\begin{array}{l}\text { Manejo } \\
\text { médico }\end{array}$ & $\begin{array}{l}\text { Desenlace } \\
\text { primario }\end{array}$ & Conclusión \\
\hline $\begin{array}{l}\text { Device closure of } \\
\text { patent foramen } \\
\text { ovale versus } \\
\text { medical therapy } \\
\text { in cryptogenic } \\
\text { stroke. A } \\
\text { systematic } \\
\text { review and } \\
\text { meta-analysis. J } \\
\text { Am Coll Cardiol } \\
\text { Intv } \\
2013 ; 6: 1316-23^{23}\end{array}$ & $\begin{array}{l}\text { Metaanálisis de } \\
\text { estudios } \\
\text { controlados } \\
\text { comparando el } \\
\text { cierre con } \\
\text { dispositivo y la } \\
\text { terapia médica } \\
\text { en la } \\
\text { prevención de } \\
\text { eventos } \\
\text { neurológicos } \\
\text { recurrentes en } \\
\text { pacientes con } \\
\text { ECV } \\
\text { criptogénico y } \\
\text { foramen oval } \\
\text { permeable. } \\
\text { Incluye Respect } \\
\text { Trial, PC Trial, } \\
\text { Clossure I Trial. }\end{array}$ & 2303 & 45,7 años & $52,7 \%$ & $33,9 \%$ & 2,9 años & $\begin{array}{l}\text { Ver } \\
\text { descripción } \\
\text { de cada uno } \\
\text { de los } \\
\text { estudios }\end{array}$ & $\begin{array}{l}\text { Ver } \\
\text { descripción } \\
\text { de cada uno } \\
\text { de los } \\
\text { estudios }\end{array}$ & $\begin{array}{l}\text { Si el cierre } \\
\text { percutáneo } \\
\text { del foramen } \\
\text { oval } \\
\text { permeable } \\
\text { es superior } \\
\text { a la terapia } \\
\text { médica en } \\
\text { pacientes } \\
\text { con ECV } \\
\text { Criptogé- } \\
\text { nico }\end{array}$ & $\begin{array}{l}\text { Hay un } 33 \% \text { a } 39 \% \\
\text { de reducción en } \\
\text { eventos } \\
\text { neurológicos } \\
\text { recurrentes } \\
\text { dependiendo de la } \\
\text { población } \\
\text { analizada. Este } \\
\text { Metanálisis sugiere } \\
\text { que el cierre } \\
\text { percutáneo del } \\
\text { foramen oval } \\
\text { permeable con } \\
\text { dispositivo es } \\
\text { benéfico } \\
\text { comparado con la } \\
\text { terapia médica en } \\
\text { la prevención de } \\
\text { ECV Criptogénico } \\
\text { recurrente. }\end{array}$ \\
\hline Estudio & Cohorte & $\begin{array}{l}\text { No. } \\
\text { Ptes }\end{array}$ & $\begin{array}{l}\text { Edad } \\
\text { promedio }\end{array}$ & Hombres & $\begin{array}{l}\text { Aneurisma } \\
\text { Septum }\end{array}$ & $\begin{array}{l}\text { Años } \\
\text { seguimiento }\end{array}$ & Intervención & $\begin{array}{l}\text { Manejo } \\
\text { médico }\end{array}$ & $\begin{array}{l}\text { Desenlace } \\
\text { primario }\end{array}$ & Conclusión \\
\hline $\begin{array}{l}\text { Is Transcatheter } \\
\text { Closure Better } \\
\text { than Medical } \\
\text { therapy for } \\
\text { cryptogenic } \\
\text { stroke with } \\
\text { patent foramen } \\
\text { ovale? A } \\
\text { meta-analysis of } \\
\text { randomised } \\
\text { trials. Heart, } \\
\text { Lung and } \\
\text { Circulation. } \\
\text { 2013;22:903-90910 }\end{array}$ & $\begin{array}{l}\text { Metaanálisis } \\
\text { Que incluye } \\
\text { RESPECT trial, } \\
\text { PC trial, } \\
\text { CLOSSURE I } \\
\text { trial }\end{array}$ & 2303 & 45,7 años & $52,7 \%$ & $33,9 \%$ & 2,9 años & $\begin{array}{l}\text { Ver } \\
\text { descripción } \\
\text { de cada uno } \\
\text { de los } \\
\text { estudios }\end{array}$ & $\begin{array}{l}\text { Ver } \\
\text { descripción } \\
\text { de cada uno } \\
\text { de los } \\
\text { estudios }\end{array}$ & $\begin{array}{l}\mathrm{ECV}, \mathrm{AIT} \text {, } \\
\text { muerte }\end{array}$ & $\begin{array}{l}\text { En pacientes con } \\
\text { ECV Criptogénico } \\
\text { o AIT con foramen } \\
\text { oval permeable, el } \\
\text { cierre con } \\
\text { dispositivo no } \\
\text { confiere ventaja } \\
\text { sobre la terapia } \\
\text { médica y es } \\
\text { asociado con } \\
\text { eventos adversos } \\
\text { como } \\
\text { complicación } \\
\text { vascular y } \\
\text { fibrilación } \\
\text { auricular. }\end{array}$ \\
\hline
\end{tabular}




\begin{tabular}{|c|c|c|c|c|c|c|c|c|c|c|}
\hline Estudio & Cohorte & $\begin{array}{l}\text { No. } \\
\text { Ptes }\end{array}$ & $\begin{array}{l}\text { Edad } \\
\text { promedio }\end{array}$ & Hombres & $\begin{array}{l}\text { Aneurisma } \\
\text { Septum }\end{array}$ & $\begin{array}{l}\text { Años } \\
\text { seguimiento }\end{array}$ & Intervención & $\begin{array}{l}\text { Manejo } \\
\text { médico }\end{array}$ & $\begin{array}{l}\text { Desenlace } \\
\text { primario }\end{array}$ & Conclusión \\
\hline $\begin{array}{l}\text { Patent foramen } \\
\text { ovale closure vs } \\
\text { medical therapy } \\
\text { for stroke } \\
\text { prevention: } \\
\text { meta-analysis of } \\
\text { randomized } \\
\text { trials and } \\
\text { review of } \\
\text { heterogeneity in } \\
\text { meta-analyses. } \\
\text { Canadian } \\
\text { Journal of } \\
\text { Cardiology. } \\
2014 ; 30: 1216- \\
24^{27}\end{array}$ & $\begin{array}{l}\text { Metaanálisis } \\
\text { que incluye } \\
\text { RESPECT trial, } \\
\text { PC trial, } \\
\text { CLOSSURE trial }\end{array}$ & 2303 & 45,7 años & $52,7 \%$ & $33,9 \%$ & 2,9 años & $\begin{array}{l}\text { Ver } \\
\text { descripción } \\
\text { de cada uno } \\
\text { de los } \\
\text { estudios }\end{array}$ & $\begin{array}{l}\text { Ver } \\
\text { descripción } \\
\text { de cada uno } \\
\text { de los } \\
\text { estudios }\end{array}$ & $\begin{array}{l}\text { El desenlace } \\
\text { primario de } \\
\text { este análisis } \\
\text { fue un } \\
\text { Compuesto } \\
\text { de ECV o AIT }\end{array}$ & $\begin{array}{l}\text { En pacientes con } \\
\text { ECV Criptogénico } \\
\text { el cierre } \\
\text { transcatéter del } \\
\text { foramen oval } \\
\text { permeable no } \\
\text { reduce } \\
\text { significativamente } \\
\text { a corto plazo la } \\
\text { incidencia de ECV } \\
\text { recurrente o AIT } \\
\text { comparado con la } \\
\text { terapia } \\
\text { antitrombótica. En } \\
\text { contraste el cierre } \\
\text { de foramen oval } \\
\text { permeable } \\
\text { incremento la } \\
\text { fibrilación } \\
\text { auricular. }\end{array}$ \\
\hline $\begin{array}{l}\text { Transcatheter } \\
\text { patent foramen } \\
\text { ovale closure } \\
\text { versus medical } \\
\text { therapy for } \\
\text { cryptogenic } \\
\text { stroke: a } \\
\text { meta-analysis of } \\
\text { randomized } \\
\text { clinical trials. } \\
\text { Riaz et al. BMC } \\
\text { Cardiovascular } \\
\text { Disorders. } \\
\text { 2013;13:116 } \\
\text { 38 }\end{array}$ & $\begin{array}{l}\text { Metaanálisis } \\
\text { que incluye } \\
\text { RESPECT trial, } \\
\text { PC trial, } \\
\text { CLOSSURE trial }\end{array}$ & 2303 & 45,7 años & $52,7 \%$ & $33,9 \%$ & 2,9 años & $\begin{array}{l}\text { Ver } \\
\text { descripción } \\
\text { de cada uno } \\
\text { de los } \\
\text { estudios }\end{array}$ & $\begin{array}{l}\text { Ver } \\
\text { descripción } \\
\text { de cada uno } \\
\text { de los } \\
\text { estudios }\end{array}$ & $\begin{array}{l}\text { El desenlace } \\
\text { primario fue } \\
\text { un } \\
\text { Compuesto } \\
\text { de muerte, } \\
\text { ECV y AIT. }\end{array}$ & $\begin{array}{l}\text { En este } \\
\text { metanálisis con } \\
\text { intención a tratar } \\
\text { el cierre del } \\
\text { foramen oval } \\
\text { permeable con } \\
\text { dispositivo no fue } \\
\text { superior a la } \\
\text { terapia médica } \\
\text { convencional en la } \\
\text { prevención } \\
\text { secundaria de ECV } \\
\text { Criptogénico. }\end{array}$ \\
\hline
\end{tabular}




\begin{tabular}{|c|c|c|c|c|c|c|c|c|c|c|}
\hline Estudio & Cohorte & $\mathrm{n}$ & $\begin{array}{l}\text { Edad } \\
\text { promedio }\end{array}$ & Hombres & $\begin{array}{l}\text { Aneurisma } \\
\text { Septum }\end{array}$ & $\begin{array}{l}\text { Años } \\
\text { seguimiento }\end{array}$ & Intervención & $\begin{array}{l}\text { Manejo } \\
\text { médico }\end{array}$ & $\begin{array}{l}\text { Desenlace } \\
\text { primario } \\
\end{array}$ & Conclusión \\
\hline $\begin{array}{l}\text { Safety and } \\
\text { efficacy of } \\
\text { device closure } \\
\text { for patent } \\
\text { foramen ovale } \\
\text { for secondary } \\
\text { prevention of } \\
\text { neurological } \\
\text { events: } \\
\text { Comprehensive } \\
\text { systematic } \\
\text { review and } \\
\text { meta-analysis of } \\
\text { randomized } \\
\text { controlled } \\
\text { trials. } \\
\text { Cardiovascular } \\
\text { Revasculariza- } \\
\text { tion Medicine } 14 \\
\text { (2013) 349-355. } \\
\text { (36) }\end{array}$ & $\begin{array}{l}\text { metaanálisis } \\
\text { que incluye } \\
\text { Respect Trial, } \\
\text { PC Trial, } \\
\text { Clossure Trial }\end{array}$ & 2303 & 45,7 años & $52,7 \%$ & $33,9 \%$ & 2,9 años & $\begin{array}{l}\text { Ver } \\
\text { descripción } \\
\text { de cada uno } \\
\text { de los } \\
\text { estudios }\end{array}$ & $\begin{array}{l}\text { Ver } \\
\text { descripción } \\
\text { de cada uno } \\
\text { de los } \\
\text { estudios }\end{array}$ & $\begin{array}{l}\text { Compuesto } \\
\text { de ECV, } \\
\text { muerte o } \\
\text { ECV fatal. }\end{array}$ & $\begin{array}{l}\text { No hay } \\
\text { diferencia esta- } \\
\text { dísticamente } \\
\text { significativa en } \\
\text { comparación a } \\
\text { la terapia } \\
\text { médica. Hubo } \\
\text { una tendencia } \\
\text { a mejores } \\
\text { desenlaces en } \\
\text { el grupo de } \\
\text { cierre del } \\
\text { foramen oval } \\
\text { permeable. } \\
\text { Parece que los } \\
\text { hombres se } \\
\text { pueden } \\
\text { beneficiar más } \\
\text { del cierre } \\
\text { percutáneo que } \\
\text { con la terapia } \\
\text { médica. }\end{array}$ \\
\hline $\begin{array}{l}\text { Percutaneous } \\
\text { closure of } \\
\text { patent foramen } \\
\text { ovale for } \\
\text { cryptogenic } \\
\text { stroke: A } \\
\text { meta-analysis of } \\
\text { randomized } \\
\text { controlled } \\
\text { trials. } \\
\text { International } \\
\text { Journal of } \\
\text { Cardiology. } \\
\text { 2013;168:4132-8 }\end{array}$ & $\begin{array}{l}\text { Metaanálisis } \\
\text { que incluye } \\
\text { RESPECT trial, } \\
\text { PC trial, } \\
\text { CLOSSURE trial }\end{array}$ & 2303 & 45,7 años & $52,7 \%$ & $33,9 \%$ & 2,9 años & $\begin{array}{l}\text { Ver } \\
\text { descripción } \\
\text { de cada uno } \\
\text { de los } \\
\text { estudios }\end{array}$ & $\begin{array}{l}\text { Ver } \\
\text { descripción } \\
\text { de cada uno } \\
\text { de los } \\
\text { estudios }\end{array}$ & $\begin{array}{l}\text { ECV, AIT y } \\
\text { todas las } \\
\text { causas de } \\
\text { muerte }\end{array}$ & $\begin{array}{l}\text { No hubo } \\
\text { diferencia } \\
\text { significativa } \\
\text { entre los } \\
\text { grupos. }\end{array}$ \\
\hline
\end{tabular}




\begin{tabular}{|c|c|c|c|c|c|c|c|c|c|c|}
\hline Estudio & Cohorte & $\mathrm{n}$ & $\begin{array}{l}\text { Edad } \\
\text { promedio }\end{array}$ & Hombres & $\begin{array}{l}\text { Aneurisma } \\
\text { Septum }\end{array}$ & $\begin{array}{l}\text { Años } \\
\text { seguimiento }\end{array}$ & Intervención & $\begin{array}{l}\text { Manejo } \\
\text { médico }\end{array}$ & $\begin{array}{l}\text { Desenlace } \\
\text { primario }\end{array}$ & Conclusión \\
\hline $\begin{array}{l}\text { Percutaneous } \\
\text { Closure versus } \\
\text { Medical Therapy } \\
\text { alone for } \\
\text { cryptogenic } \\
\text { stroke patients } \\
\text { with a patent } \\
\text { foramen ovale: } \\
\text { meta-analysis of } \\
\text { randomized } \\
\text { controlled } \\
\text { trials. Tex Heart } \\
\text { Inst J. } \\
\text { 2014;41(4): } \\
357-67^{31}\end{array}$ & $\begin{array}{l}\text { Metaanálisis } \\
\text { que incluye } \\
\text { RESPECT trial, } \\
\text { PC trial, } \\
\text { CLOSSURE trial }\end{array}$ & 2303 & 45,7 años & $52,7 \%$ & $33,9 \%$ & 2,9 años & $\begin{array}{l}\text { Ver } \\
\text { descripción } \\
\text { de cada uno } \\
\text { de los } \\
\text { estudios }\end{array}$ & $\begin{array}{l}\text { Ver } \\
\text { descripción } \\
\text { de cada uno } \\
\text { de los } \\
\text { estudios }\end{array}$ & $\begin{array}{l}\text { Combinado } \\
\text { de muerte, } \\
\text { ECV y AIT }\end{array}$ & $\begin{array}{l}\text { Cuando se } \\
\text { comparó con la } \\
\text { terapia médica } \\
\text { sola, el cierre } \\
\text { del foramen } \\
\text { oval permeable } \\
\text { con la terapia } \\
\text { médica } \\
\text { demostró una } \\
\text { tendencia a la } \\
\text { disminución de } \\
\text { eventos } \\
\text { combinados. }\end{array}$ \\
\hline Estudio & Cohorte & nPtes & $\begin{array}{l}\text { Edad } \\
\text { promedio }\end{array}$ & Hombres & $\begin{array}{l}\text { Aneurisma } \\
\text { Septum }\end{array}$ & $\begin{array}{l}\text { Años } \\
\text { seguimiento }\end{array}$ & Intervención & $\begin{array}{l}\text { Manejo } \\
\text { médico }\end{array}$ & $\begin{array}{l}\text { Desenlace } \\
\text { primario }\end{array}$ & Conclusión \\
\hline $\begin{array}{l}\text { Percutaneous } \\
\text { Closure of } \\
\text { Patent Foramen } \\
\text { Ovale in } \\
\text { Cryptogenic } \\
\text { Embolism. N } \\
\text { Engl J Med 2013; } \\
\text { 368:1083-91. PC } \\
\text { TRIAL. }\end{array}$ & $\begin{array}{l}\text { Multicenter, } \\
\text { superiority trial } \\
\text { in } 29 \text { centers in } \\
\text { Europe, } \\
\text { Canada, Brazil, } \\
\text { and Australia }\end{array}$ & 414 & 44 & $49.8 \%$ & $23,7 \%$ & 4,1 & $\begin{array}{l}\text { Amplatzer }{ }^{\circledR} \\
\text { y } \\
100-325 \mathrm{mg} \\
\text { Aspirina por } \\
6 \text { meses y } \\
75-150 \mathrm{mg} \\
\text { clopidogrel } \\
\text { por } 1-6 \\
\text { meses }\end{array}$ & $\begin{array}{l}\text { Al menos } 1 \\
\text { terapia anti- } \\
\text { trombótica }\end{array}$ & $\begin{array}{l}\text { Compuesto } \\
\text { de muerte } \\
\text { de cualquier } \\
\text { causa, ECV } \\
\text { no fatal, AIT } \\
\text { y embolismo } \\
\text { periférico. }\end{array}$ & $\begin{array}{l}\text { El cierre de un } \\
\text { foramen oval } \\
\text { permeable para } \\
\text { prevención } \\
\text { secundaria de } \\
\text { embolismo } \\
\text { Criptogénico no } \\
\text { resulta en } \\
\text { reducción } \\
\text { significativa del } \\
\text { riesgo de } \\
\text { evento } \\
\text { embólico } \\
\text { recurrente o } \\
\text { muerte } \\
\text { comparado con } \\
\text { la terapia } \\
\text { médica. }\end{array}$ \\
\hline
\end{tabular}




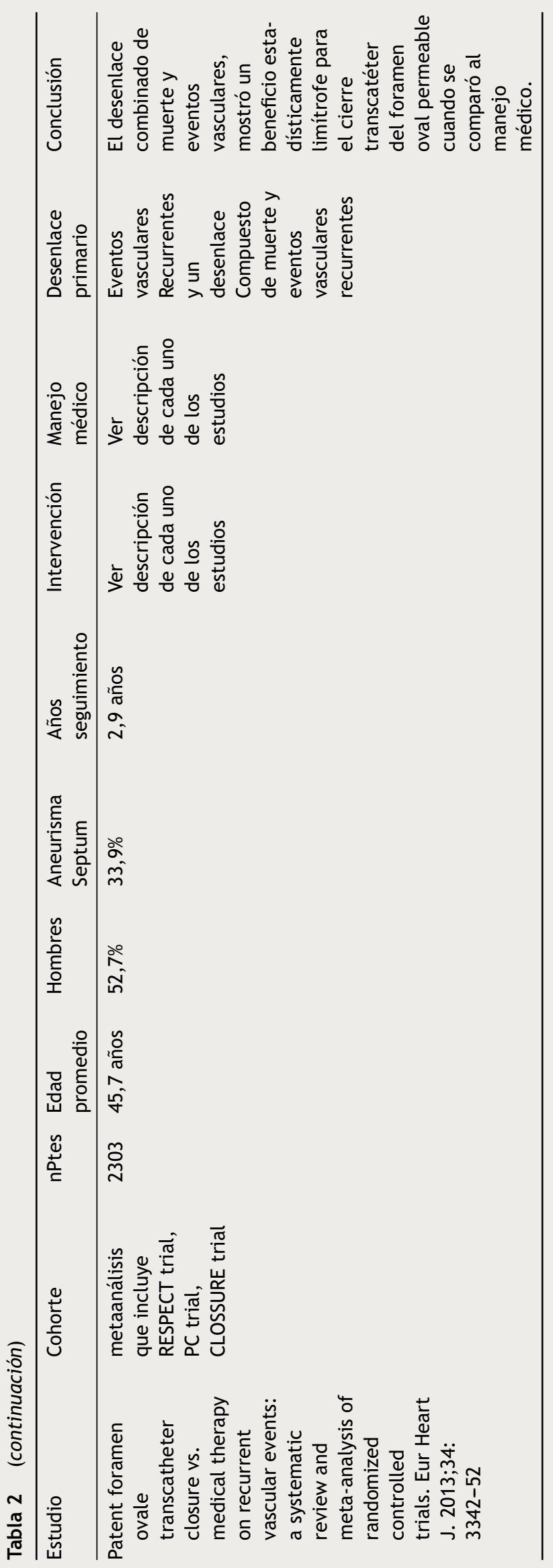

De otro lado, en 2013 Meier $^{20}$, presentó el PC trial, ensayo clínico, multicéntrico, aleatorizado, en el que participaron 29 centros en Europa, Canadá, Brasil y Australia, que reclutó pacientes entre el año 2000 a 2009, y un total de 414 pacientes, 204 de ellos aleatorizados a cierre con dispositivo (Amplatzer PFO Occluder ${ }^{\circledR}$ ) y 210 a terapia médica. Los pacientes incluidos eran menores de 60 años, con foramen oval permeable documentado mediante ecocardiograma trasnesofágico y ataque cerebrovascular de origen criptogénico; se excluyeron pacientes con otras causas de ataque cerebrovascular. La terapia antitrombótica permitida en el grupo de cierre percutáneo fue aspirina $100-325 \mathrm{mg} /$ día por 6 meses o ticlopidina $250-500 \mathrm{mg} /$ día o clopidogrel $75 \mathrm{mg}$ de 1 a 6 meses. En el grupo de terapia médica, la decisión de terapia antitrombótica quedó a discreción del grupo tratante. El desenlace primario fue un punto final compuesto que incluyó muerte, ataque cerebrovascular no fatal, accidente isquémico transitorio o embolia periférica. Se logró implante del dispositivo en el 95,9\% de los casos, con un seguimiento promedio en el estudio de 4,1 años. Respecto a los resultados, el punto final primario se encontró en el $3,4 \%$ de los pacientes en el grupo asignado a dispositivo vs. $5,2 \%$ en el grupo asignado a terapia médica (HR: 0,63; IC 95: 0,24-1,62; $p=0,34$ ); ataque cerebrovascular $0,5 \%$ en el grupo asignado a dispositivo vs. $2,4 \%$ en el grupo asignado a terapia médica (HR: 0,20 IC 95; 0,02-1,72; $p=0,14)$. Respecto a los eventos adversos, estos se presentaron en el $34,8 \%$ de los pacientes en el grupo asignado a dispositivo vs. $29,5 \%$ en el grupo asignado a terapia médica sin diferencias significativas entre ambos grupos. Concluyeron que no se encontró una reducción del riesgo de eventos recurrentes de ataque cerebrovascular o accidente isquémico transitorio en el grupo de cierre con dispositivo en comparación con el grupo de terapia médica.

Los tres estudios previamente descritos, son los pivote de varios metaanálisis y revisiones sistemáticas ${ }^{10,23,27,28,30,31,36,38}$, que en conclusión, no muestran beneficios del cierre con dispositivo (diferentes tipos de dispositivo) en comparación con la terapia médica en la reducción del riesgo de nuevos eventos de ataque cerebrovascular, accidente isquémico transitorio y muerte, principalmente; esta información se resume en la tabla 2. Por esta razón y con base en la información existente, se decidió no proceder a efectuar un metaanálisis de la información dado que no existe un beneficio real en su realización.

\section{Discusión}

Los ensayos clínicos incluidos en este trabajo ${ }^{18-20}$, son la base de la evidencia disponible para el cierre percutáneo con dispositivo en pacientes con ataque cerebrovascular isquémico criptogénico y foramen oval permeable. Como puntos relevantes para discusión se encontró que el perfil de riesgo de la población es relativamente similar en los tres estudios; sin embargo, las definiciones operativas de los desenlaces y el cálculo del tamaño de muestra, en especial lo concerniente al poder del estudio y tasa de eventos esperadas, son discretamente diferentes. Existen dos tipos de dispositivos en evaluación (Amplatzer ${ }^{\circledR}$ y Starflex ${ }^{\circledR}$ ), los cuales tienen un éxito aceptable de implante con un número significativo de 
complicaciones relacionadas con lesiones vasculares, taponamiento cardiaco y la presencia de fibrilación auricular, que si bien, no son significativamente diferentes en comparación con el grupo de terapia médica en los estudios, la aparición en los primeros 30 días posterior al procedimiento de fibrilación auricular en un porcentaje superior y estadísticamente significativo al grupo control, no es una condición despreciable.

Las estrategias antitrombóticas confieren un sesgo importante en la evaluación de los datos, puesto que son variables tanto en el grupo asignado a dispositivo como en el grupo asignado a terapia médica; en este último, cabe resaltar que no existe diferencia en los resultados, según el tipo de estrategia antitrombótica elegida.

La tasa de eventos recurrentes de ataque cerebrovascular o accidente isquémico transitorio en los tres estudios es menor al $5 \%$, porcentaje bajo en comparación con diferentes condiciones médicas que se asocian con aumento del riesgo de eventos, incluso de aquellos relacionados con el control pobre de factores de riesgo cardiovascular tradicionales.

La realización de subestudios y subanálisis con las poblaciones descritas en los tres estudios pivote, han sugerido la presencia de un beneficio discreto a favor del cierre con dispositivo en pacientes con alto riesgo de recurrencia, ataque cerebrovascular grande o shunt significativo; pese a ello, la heterogeneidad de los datos, limita la extrapolación de la información a las poblaciones en las cuales fueron diseñados los estudios primarios.

Cabe recordar que los costos relacionados con el procedimiento y los eventos adversos esperables, sin un beneficio claro en la reducción del riesgo, son un factor fundamental en la toma de decisiones en la práctica médica diaria, consideración que deberá tenerse en cuenta para la práctica de este tipo de procedimientos en Colombia.

\section{Conclusión}

En pacientes adultos con ataque cerebrovascular isquémico o accidente isquémico transitorio de origen criptogénico, en quienes se ha documentado mediante ecocardiografía transesofágica la presencia de foramen oval permeable, no existe beneficio en la reducción del riesgo de recurrencia de ataque cerebrovascular, accidente isquémico transitorio o eventos neurológicos en comparación con la terapia medica convencional. Subanálisis de los tres estudios pivote, sugieren la presencia de un beneficio discreto a favor del cierre con dispositivo en pacientes con alto riesgo de recurrencia, ataque cerebrovascular grande o shunt significativo. No obstante, la heterogeneidad de los datos, limita la extrapolación de la información a las poblaciones en las cuales fueron diseñados los estudios primarios.

\section{Responsabilidades éticas}

Protección de personas y animales. Los autores declaran que para esta investigación no se han realizado experimentos en seres humanos ni en animales.

Confidencialidad de los datos. Los autores declaran que en este artículo no aparecen datos de pacientes.
Derecho a la privacidad y consentimiento informado. Los autores declaran que en este artículo no aparecen datos de pacientes.

\section{Bibliografía}

1. Hagen PT, Scholz DG, Edwards WD. Incidence and size of patent foramen ovale during the first 10 decades of life: an autopsy study of 965 normal hearts. Mayo Clin Proc. 1984;59(1): 17-20.

2. Di Tullio MR, Jin Z, Russo C, Elkind MSV, Rundek T, Yoshita $M$, et al. Patent foramen ovale, subclinical cerebrovascular disease, and ischemic stroke in a population-based cohort. J Am Coll Cardiol. 2013;62(1):35-41.

3. Wessler BS, Thaler DE, Ruthazer R, Weimar C, Di Tullio MR, Elkind MSV, et al. Transesophageal echocardiography in cryptogenic stroke and patent foramen ovale: analysis of putative high-risk features from the risk of paradoxical embolism database. Circ Cardiovasc Imaging. 2014;7(1):125-31.

4. Kent DM, Thaler DE. The Risk of Paradoxical Embolism (RoPE) Study: developing risk models for application to ongoing randomized trials of percutaneous patent foramen ovale closure for cryptogenic stroke. Trials. 2011;12:185.

5. Agarwal S, Bajaj NS, Kumbhani DJ, Tuzcu EM, Kapadia SR. Metaanalysis of transcatheter closure versus medical therapy for patent foramen ovale in prevention of recurrent neurological events after presumed paradoxical embolism. JACC Cardiovasc Interv. 2012;5(7):777-89.

6. Almekhlafi MA, Wilton SB, Rabi DM, Ghali WA, Lorenzetti DL, Hill MD. Recurrent cerebral ischemia in medically treated patent foramen ovale: a meta-analysis. Neurology. 2009;73(2):89-97.

7. Kitsios GD, Lasker A, Singh J, Thaler DE. Recurrent stroke on imaging and presumed paradoxical embolism: a cross-sectional analysis. Neurology. 2012;78(13):993-7.

8. Wahl A, Jüni P, Mono M-L, Kalesan B, Praz F, Geister L, et al. Long-term propensity score-matched comparison of percutaneous closure of patent foramen ovale with medical treatment after paradoxical embolism. Circulation. 2012;125(6):803-12.

9. Ntaios G, Papavasileiou V, Makaritsis K, Michel P. PFO closure vs. medical therapy in cryptogenic stroke or transient ischemic attack: A systematic review and meta-analysis. Int J Cardiol. 2013;169(2):101-5.

10. Nagaraja V, Raval J, Eslick GD, Burgess D, Denniss AR. Is transcatheter closure better than medical therapy for cryptogenic stroke with patent foramen ovale? A meta-analysis of randomised trials. Heart Lung Circ. Australian and New Zealand Society of Cardiac and Thoracic Surgeons (ANZSCTS) and the Cardiac Society of Australia and New Zealand (CSANZ). 2013;22(11):903-9.

11. Kutty S, Sengupta PP, Khandheria BK. Patent foramen ovale: the known and the to be known. J Am Coll Cardiol. 2012;59(19):1665-71.

12. FW Z. Thrombose de plusieurs branches de la veine cave inferieure avec embolies consecutives das les arteres pulmonair, slenique, renale et iliaque droite. Rev Med Suisse Rom. $1881 ; 1: 227-37$.

13. Holmes DR, Cohen H, Katz WE, Reeder GS. Patent foramen ovale, systemic embolization and closure. Curr Probl Cardiol. 2004;29(2):56-94.

14. Roger VL, Go AS, Lloyd-Jones DM, Adams RJ, Berry JD, Brown TM, et al. Heart disease and stroke statistics-2011 update: a report from the American Heart Association. Circulation. 2011;123(4):e18-209.

15. Lechat P, Mas JL, Lascault G, Loron P, Theard M, Klimczac M, et al. Prevalence of patent foramen ovale in patients with stroke. N Engl J Med. 1988;318(18):1148-52. 
16. Holmes DR, Cohen HA, Ruiz C. Patent foramen ovale, systemic embolization, and closure. Curr Probl Cardiol. 2009;34(10):483-530.

17. Hanley PC, Tajik AJ, Hynes JK, Edwards WD, Reeder GS, Hagler DJ, et al. Diagnosis and classification of atrial septal aneurysm by two-dimensional echocardiography: report of 80 consecutive cases. J Am Coll Cardiol. 1985;6(6):1370-82.

18. Furlan AJ, Reisman M, Massaro J, Mauri L, Adams H, Albers GW, et al. Closure or medical therapy for cryptogenic stroke with patent foramen ovale. N Engl J Med. 2012;366(11):991-9.

19. Carroll JD, Saver JL, Thaler DE, Smalling RW, Berry S, MacDonald LA, et al. Closure of patent foramen ovale versus medical therapy after cryptogenic stroke. N Engl J Med. 2013;368(12):1092-100.

20. Meier B, Kalesan B, Mattle HP, Khattab AA, Hildick-Smith D, Dudek $D$, et al. Percutaneous closure of patent foramen ovale in cryptogenic embolism. N Engl J Med. 2013;368(12):1083-91.

21. Graves RS. Users' Guides to the Medical Literature: A Manual for Evidence-Based Clinical Practice. Journal of the Medical Library Association. Medical Library Association. 2002:483.

22. Mügge A, Daniel WG, Angermann C, Engberding R. Atrial septal aneurysm in adult patients. Circulation. 1993:2785-92.

23. Khan AR, Bin Abdulhak AA, Sheikh MA, Khan S, Erwin PJ, Tleyjeh I, et al. Device closure of patent foramen ovale versus medical therapy in cryptogenic stroke: A systematic review and metaanalysis. JACC Cardiovasc Interv. 2013;6(12):1316-23.

24. Von Bardeleben RS, Richter C, Otto J, Himmrich L, Schnabel R, Kampmann C, et al. Long term follow up after percutaneous closure of PFO in 357 patients with paradoxical embolism: Difference in occlusion systems and influence of atrial septum aneurysm. Int J Cardiol. 2009;134(1):33-41.

25. Hornung M, Bertog SC, Franke J, Id D, Taaffe M, Wunderlich N, et al. Long-term results of a randomized trial comparing three different devices for percutaneous closure of a patent foramen ovale. Eur Heart J. 2013;34(43):3362-9.

26. Patti G, Pelliccia F, Gaudio C, Greco C. Meta-analysis of net longterm benefit of different therapeutic strategies in patients with cryptogenic stroke and patent foramen ovale. Am J Cardiol. 2015;115(6):837-43.

27. Udell JA, Opotowsky AR, Khairy P, Silversides CK, Gladstone DJ, O'Gara PT, et al. Patent foramen ovale closure versus medical therapy for stroke prevention: meta analysis of randomized trials and review of heterogeneity in meta analyses. Can J Cardiol. Canadian Cardiovascular Society. 2014;30(10):1216-24.

28. Rengifo-Moreno P, Palacios IF, Junpaparp P, Witzke CF, Morris DL, Romero-Corral A. Patent foramen ovale transcatheter closure vs. medical therapy on recurrent vascular events: a systematic review and meta-analysis of randomized controlled trials. Eur Heart J. 2013;34(43):3342-52.

29. Davis D, Gregson J, Willeit P, Stephan B, Al-Shahi Salman R, Brayne C. Patent foramen ovale, ischemic stroke and migraine: systematic review and stratified meta-analysis of association studies. Neuroepidemiology. 2013;40(1):56-67.

30. Kwong JSW, Lam Y-Y, Yu C-M. Percutaneous closure of patent foramen ovale for cryptogenic stroke: a meta-analysis of randomized controlled trials. Int J Cardiol. 2013;168(4):4132-8.

31. Pickett CA, Villines TC, Ferguson MA, Hulten EA. Percutaneous closure versus medical therapy alone. 2013;128:15-9.

32. Wöhrle J, Bertrand B, Soøndergaard L, Turner M, Scholtz W, Ibrahim R, et al. PFO closuRE and Cryptogenlc StrokE (PRECISE) registry: A multi-center, international registry. Clin Res Cardiol. 2012;101(10):787-93.

33. Furlan AJ. PFO closure: CLOSURE. Stroke. 2013;44 suppl.1: 2013-6.

34. Collection D. Recurrent cerebrovascular events associated with patent. New Eng J Med. 2001;345(24):1740-6.

35. Ma B, Liu G, Chen X, Zhang J, Liu Y, Shi J. Risk of stroke in patients with patent foramen ovale: an updated metaanalysis of observational studies. J Stroke Cerebrovasc Dis. 2014;23(5):1-9.

36. Hakeem A, Marmagkiolis K, Hacioglu Y, Uretsky BF, Gundogdu $B$, Leesar M, et al. Safety and efficacy of device closure for patent foramen ovale for secondary prevention of neurological events: Comprehensive systematic review and meta-analysis of randomized controlled trials. Cardiovasc Revasc Med. 2013;14(6):349-55.

37. Abaci A, Unlu S, Alsancak Y, Kaya U, Sezenoz B. Short and long term complications of device closure of atrial septal defect and patent foramen ovale: Meta-analysis of 28,142 patients from 203 studies. Catheter Cardiovasc Interv. 2013;82(7):1123-38.

38. Bin Riaz I, Dhoble A, Mizyed A, Hsu C-H, Husnain M, Lee JZ, et al. Transcatheter patent foramen ovale closure versus medical therapy for cryptogenic stroke: a meta-analysis of randomized clinical trials. BMC Cardiovasc Disord. 2013;13:116.

39. McGrath ER, Paikin JS, Motlagh B, Salehian O, Kapral MK, O'Donnell MJ. Transesophageal echocardiography in patients with cryptogenic ischemic stroke: A systematic review. Am Heart J. 2014;168(5), 706-12e14.

40. Komar M, Podolec P, Przewłocki T, Wilkotek P, Tomkiewicz-Pajạk L, Motyl R. Transoesophageal echocardiography can help distinguish between patients with symptomatic and asymptomatic patent foramen ovale. Kardiol Pol. 2012;70(12):1258-63.

41. Capodanno D, Milazzo G, Vitale L, Di Stefano D, Di Salvo M, Grasso C, et al. Updating the evidence on patent foramen ovale closure versus medical therapy in patients with cryptogenic stroke: a systematic review and comprehensive meta-analysis of 2,303 patients from three randomised trials and 2,231 patients from 11 observational. Euro Intervention. 2014;9(11):1342-9.

42. Wolfrum M, Froehlich GM, Knapp G, Casaubon LK, DiNicolantonio JJ, Lansky AJ, et al. Stroke prevention by percutaneous closure of patent foramen ovale: a systematic review and metaanalysis. Heart. 2014;100(5):389-95. 